Adalbert de VOGÜÉ OSB

(La Pierre-qui-Vivre. Abbaye Sainte-Marie)

\title{
LA JOIE DE L'ESPRIT SAINT CHEZ SAINT BENOÎT
}

C'est un fait singulier que la joie (gaudium) n'apparaît guère, dans la Règle de saint Benoît, qu'en deux passages où il est question de mortifications ou d'épreuves. Si l'on met à part une phrase du directoire de l'abbé, où Benoît souhaite à celui-ci „d'éprouver la joie de voir grandir son troupeau", ${ }^{\text {, les autres }}$ occurrences du substantif gaudium et du verbe gaudere rendent un son différent et inattendu.

1. La joie du moine dans l'épreuve. Au chapitre de l'humilité, d'abord, dans ce „degré d'humilité” particulièrement long et pathétique qu'est le quatrième le moine y est affronté ,à des choses dures et contrariantes, voire à toute sorte de torts qu'on lui inflige" - Benoît cite un verset de psaume où des malheureux se plaignent d'être traités „comme des brebis de boucherie”, et il ajoute:

„Avec l'assurance que leur donne l'espoir d'une récompense divine, ils poursuivent joyeusemenf en disant: «Toutes ces épreuves, nous les surmontons à cause de Celui qui nous a aimés»"².

Cette note de joie (gaudentes) au milieu de l'épreuve retentit de nouveau dans le chapitre du carême. Elle s'y fait même entendre deux fois de suite. D'abord Benoît invite chaque moine à „offrir" au Seigneur, en plus des retranchements communautaires, „quelque chose qui procède de sa propre volonté", et il spécifie que cette offrande spontanée et coûteuse d'un chacun se fera „dans la joie du Saint Esprit” (cum gaudio Spiritus Sancti) ${ }^{3}$. Aussitôt après, il réitère et précise cette invitation:

${ }^{1}$ Regula Benedicti [= RB] 2, 32, éd. J. Neufville, introduction, traduction et notes A. de Vogüé, SCh 181-186, Paris 1972-1977, ici 181, 450: „in augmentatione boni gregis gaudeat”.

${ }^{2}$ RB 7, 39, SCh 181, 482: „Subsequntur gaudentes et dicentes”, précédé de Ps 26, 14 et suivi de $\operatorname{Rm} 8,36$.

${ }^{3}$ RB 49, 6; SCh 182, 606: „ut unusquisque super mensuram sibi indictam aliquid propria voluntate cum gaudio Sancti Spiritus offerat Deo". 
„Que chacun retranche à son corps de la nourriture, de la boisson, du sommeil, des conversations, des plaisanteries, et qu'il attende la sainte Pâque avec la joie du désir spirituel" 4 .

Épreuves et joie, renoncements et joie: ces associations paradoxales du quatrième degré d'humilité et du chapitre sur le carême ne sont pas de pures inventions de Benoît. Dans les deux cas, un substrat scripturaire apparaît nettement. Quand on nous montre, dans le traité de l'humilité, le moine douloureux et joyeux tout ensemble, il faut prendre garde à un petit mot de trois lettres glissé par Benoît dans cette phrase: si les éprouvés surmontent joyeusement leur épreuve, c'est qu'ils ont „l'espérance” (spe) d'être récompensés par Dieu. Or l'espérance ne va pas sans la joie, dit saint Paul: „spe gaudentes". Cette parole de l'Épître aux Romains est sous-jacente au quatrième degré d'humilité .

Plus précisément encore, la ,joie du Saint Esprit” mentionnée dans le chapitre du carême rappelle un autre mot de l'Apôtre. Ecrivant aux Thessaloniciens, Paul les félicitait d'avoir „reçu la Parole, au milieu d'une grande tribulation, avec la joie de l'Esprit Saint" ${ }^{\text {. }}$ Le carême des moines italiens du $\mathrm{VI}^{\mathrm{e}}$ siècle leur fait donc revivre l'expérience des premiers chrétiens de Thessalonique, persécutés pour leur adhésion de foi au Christ: l'épreuve qu'on subit à cause de celui-ci s'accompagne de la joie de l'Esprit. Et quand Benoît, réitérant son affirmation, fait espérer que l'attente de Pâques baignera dans la ,joie du désir spirituel”, on songe non seulement au mot de 1'Épître aux Romains évoqué plus haut - spe gaudentes, ,joyeux à cause de l'espérance" mais encore aux ,fruits de l'Esprit” énumérés par le même Paul dans l'Epître aux Galates: „le fruit de l'Esprit est charité, joie, paix” et le reste (Ga 5, 22). Si la joie est qualifiée ici de „spirituelle”, c'est qu'elle est, comme l'amour, un des dons de l'Esprit Saint présent au coeur du chrétien.

La joie du moine, chez saint Benoît, est donc à la fois un concomitant de l'épreuve et un don de l'Esprit Saint. Si d'ailleurs Benoît ne prononce ce mot (gaudium) qu'en ces deux passages de sa Règle, au quatrième degré d'humilité et dans le chapitre du carême, il faut d'autant moins s'en étonner que ce temps qui précède Pâques a pour lui une valeur exemplaire: „En tout temps la vie du moine doit présenter l'observance du carême”7. La ,joie du désir spirituel”, avec laquelle on regarde vers Pâques, symbolise donc la „,convoitise spirituelle”

${ }^{4}$ RB 49, 7, SCh 182, 606: ,id est, subtrahat corpori suo de cibo, de potu, de somno, de loquacitate, de scurrilitate, et cum spiritalis desiderii gaudio sanctum Pascha expescet".

5 Cfr. RB 7, 35-43, Rm 12, 12.

${ }^{6}$ Cfr. RB 49, 6, SCh 182, 506; voir 1Th 1, 6: ,excipientes verbum in tribulatione magna cum gaudio Spiritus Sancti".

7 RB 49, 1, SCh 182, 604: „Licet omni tempore vita monachi quadragesimae debet observantiam habere". 
avec laquelle on „désire la vie éternelle”, comme dit un des Instruments des bonnes oeuvres ${ }^{8}$.

Au reste, l'extrême rareté des passages où Benoît prononce ce mot de „joie” ne doit pas faire oublier deux pages de la Règle où apparaît - sans le mot - un état d'âme analogue. L'une de ces péricopes est la fin du Prologue. Après y avoir défini le monastère, ainsi que le faisait la Règle du Maître, comme une „école du service du Seigneur”, Benoît ajoute plusieurs lignes où il formule d'abord son désir de ne pas imposer des choses pénibles, puis met en garde contre le découragement que pourraient inspirer certaines prescriptions, jugées trop rigoureuses et insupportables. Ce n'est là qu'un début, dit-il:

„La voie du salut ne peut être qu'étroite pour commencer; mais à mesure qu'on avance dans la vie religieuse et la foi, le coeur se dilate, et l'on court sur la voie des commandements de Dieu avec une indicible douceur d'amour" .

Sans prononcer le mot gaudium, Benoît évoque là un état d'âme bien proche de la joie. Celle-ci, comme dans l'échelle d'humilité et le programme du carême, résulte paradoxalement de l'épreuve elle-même: la voie étroite de l'Evangile, avec tous ses renoncements, ne resserre pas le coeur, mais l'élargit au contraire et l'épanouit. La charité divine s'empare de l'homme, le remplissant de sa douceur.

L'autre passage où apparaît quelque chose qui ressemble à la joie, est la conclusion du grand chapitre de l'humilité. Avec Cassien et le Maître, Benoît évoque là l'épanouissemnt auquel aboutit l'ascension de' l'humilité, commencée dans la crainte du Seigneur et de son jugement. A cette crainte initiale se substitue finalement l'amour. Les trois auteurs varient légèrement dans l'évocation de celui-ci - à „l'amour du bien” (Cassien) ou „de la bonne habitude” (le Maître), Benoît substitue simplement „l'amour du Christ” - mais tous trois s'accordent à parler de la „délectation des vertus” dont s'accompagne cet amour final ${ }^{10}$. Or trouver son plaisir dans l'agir vertueux, n'est-ce pas connaître une certaine joie? La joie qui épanouit l'âme est donc moins rare qu'il ne semble chez saint Benoît. Mais de façon très cohérente, elle affleure toujours dans le même contexte oblatif et sacrificiel. Plusieurs fois aussi, nous avons remarqué un substrat scripturaire évident. Cette référence au Nouveau Testament, et plus particulièrement aux écrits pauliniens, nous engage à regarder ceux-ci pour mieux comprendre ce que les écrivains monastiques en ont tiré. Voyons donc ce que saint Paul, ainsi que saint Jean, dit de la joie.

${ }^{8}$ Cfr. RB 4, 46, SCh 181, 460: „Vitam aeternam omni concupiscentia spiritali desiderare”.

9 RB Prol 48-50, SCh 181, 424: ,non ilico pavore perterritus refugias viam salutis, quae non est nisi angusto initio incipienda (cfr. Mt 7,14). Processu ver conversationis et fidei «dilatato corde» inenarrabili dilectionis dulcedine "curritur via mandatorum Dei»" (Ps 118, 32).

${ }^{10}$ Cfr. RB 7, 69, SCh 181, 490: ,amore Christi et consuetudine ipsa bona et delectatione virtutum; voir Joannes Cassianus, De institutis coenobiorum IV 39, 3; Regula Magistri 10, 90. 
2. Epreuve et joie chez saint Paul. Le chapitre de Benoît sur le carême nous a rais en présence de deux paroles de l'Apôtre qui associent l'une et l'autre la ,joie” et l',épreuve”. „Joyeux dans l'espérance, patients dans l'épreuve, persévérants dans la prière": tels seront les Romains, s'ils répondent aux voeux de saint Paul ${ }^{11}$. Et plus tôt, déjà, les Thessaloniciens étaient félicités d'avoir „reçu la Parole, au sein d'une grande épreuve, avec la joie de l'Esprit Saint" ${ }^{\prime 2}$. Cette association de la joie et de ce qui est apparemment son contraire - l'épreuve, la tribulation - se retrouvé ailleurs chez saint Paul. De sa propre personne, il disait aux Corinthiens: „Nous semblons être tristes, et nous sommes toujours joyeux" $" 13$.

D'autre part, la ,joie de l'Esprit Saint”, mentionnée dans la Lettre aux Thessaloniciens, trouve plusieurs échos dans l'épistolaire paulinien. Quand Paul énumère aux Galates les „fruits de l'Esprit”, la joie vient en second, aussitôt après l'amour, dans cette liste de neuf termes: „le fruit de l'Esprit, c'est charité, joie, paix" et le reste ${ }^{14}$. Et lorsque l'Apôtre définit, à l'usage des Romains, la véritable nature du royaume de Dieu, il écrit: „Le royaume de Dieu n'est pas nourriture et boisson, mais justice, paix et joie dans l'Esprit Saint" "15. En harmonie avec ces propos de saint Paul, les Actes des Apôtres nous montrent ses disciples en Asie Mineure, ,remplis de joie et de l'Esprit Saint", au sein même de la persécution qui se'vit contre eux ${ }^{16}$.

Une autre compagne habituelle de la joie est la prière. Après avoir, dans la phrase de l'Épître aux Romains citée plus haut, recommandé la ,joie dans l'espérance” et la „patience dans l'épreuve”, Paul ajoute aussitôt la „persévérance dans la prière" ${ }^{17}$. Et quand il dit aux Thessaloniciens: „Soyez toujours joyeux”, il ne manque pas d'ajouter: „Priez sans cesse, en toute chose rendez grâces"18. Ce dernier passage des Épîtres de Paul est d'une importance capitale pour l'histoire de la prière chrétienne. C'est en effet ce „Priez sans cesse” qui a inspiré toutes les recherches des fidèles et des communautés pour meubler le temps avec des prières: ainsi est né l'office divin des Églises et des monastères. Mais tandis que ce „Priez sans cesse” de l'Épître aux Thessaloniciens engendrait des pratiques de toute sorte et était sans cesse invoqué par les liturgistes, ruminé par les âmes pieuses, rappelé par les auteurs spirituels, les deux sentences voisines de la même Epître - „Soyez toujours joyeux” et

${ }^{11}$ Cfr. Regula magistri 12, 12: „spe gaudentes, in tribulatione patientes, orationi instantes”.

12 Cfr. 1Th 1, 6: ,,accipientes verbum in tribulatione magna cum gaudio Spiritus Sancti”. Dans cette phrase comme dans la precédénte, tribulatio („épreuve”) traduit le grec - $\theta \lambda i \psi r \varsigma$.

13 Cfr. 2 Co 6, 10: „quasi tristes, semper autem gaudentes”.

${ }^{14}$ Cfr. Ga 5, 22: „Fructus autem Spiritus est caritas, gaudium, pax”.

15 Cfr. Rm 14, 17: ,iustitia et pax et gaudium in Spiritu Sancto”.

${ }^{16}$ Cfr. Ac 13, 52: „discipuli quoque replebantur gaudio et Spiritu Sancto”.

${ }^{17}$ Cfr. Regula magistri 12, 12; voir n. 11.

${ }^{18}$ Cfr. 1Th 5, 16-18: ,semper gaudete, sine intermissione orate, in omnibus gratias agite”. 
„En toute chose rendez grâces” - ne connaissaient nullement le même développement.

Un auteur monastique célèbre, Jean Cassien, peut servir d'exemple à cet égard. Le „priez sans cesse” de l'Apôtre est cité huit fois dans ses Institutions et ses Conférences, où cette consigne néotestamentaire sert de fondement à toute l'organisation de la prière des heures ${ }^{19}$. Au contraire, la prescription voisine de saint Paul - „soyez toujours joyeux” - n'apparaît jamais, semble-t-il, dans l'oeuvre de Cassien $^{20}$. Que le moine doive prier sans cesse, on aime à le lui rappeler. Mais être toujours joyeux, on semble oublier qu'il en a aussi le devoir.

„Soyez toujours joyeux”. Un lecteur de la Règle bénédictine pourrait objecter que Benoît, comme beaucoup d'auteurs monastiques anciens, est sévère pour le rire. Un de ses, instruments des bonnes oeuvres" interdit de prononcer des ,paroles vaines et qui portent à rire”, et le suivant recommande de ,ne pas aimer le rire prolongé ou aux éclats" ${ }^{\prime 2}$. En plus de ces sentences, qui lui viennent du Maître, Benoît suit encore ce dernier, et avec lui Cassien, dans un des derniers degrés de son échelle d'humilité, qui veut qu'on ne soit pas „facile et prompt à rire" 22 . Encore ces phrasés ne condamnent-elles pas toute espèce de rire, mais seulement certaines formes de celui-ci. Mais d'autres auteurs monastiques, antérieurs ou contemporains, vont jusqu'à interdire qu'on rie, en faisant valoir non seulement le „Malheur à vous qui riez" ${ }^{23}$, mais encore l'exemple de Jésus, que l'Evangile montre parfois pleurant, jamais riant $^{24}$.

Ces mises en garde contre le rire empêchent-elles de cultiver la joie? Non, certes, car il n'est nullement nécessaire de rire pour être joyeux. C'est même l'opposé du rire - les larmes - que Benoît associe, dans son programme du carême, à la ,joie de l'Esprit Saint" qui doit dominer l'âme en cette période. Ce que le moine „offre à Dieu avec la joie de l'Esprit Saint”, c'est justement une série de retranchements qui portent non seulement sur la nourriture, la boisson et le sommeil, mais encore sur la loquacité et la plaisanterie. En même temps, la

${ }^{19}$ Voir en particulier De institutis coenobiorum I 2, 1 et Conlationes IX 3, 4.

${ }^{20}$ Cfr. l'index tres complet de M. Petschenig (CSEL 17, 408). À propos du vice de tristesse (De institutis coenobiorum 9,11), Cassien cité Ga 5,22, mais pas 1Th 5, 16. Ailleurs, il cite aussi 2Co 6,10 , mais à la fin d'une citation plus ample (2Co 6, 7-10) et sans mettre en relief le semper gaudentes, qui n'est d'ailleurs pas une consigne, mais un simple témoignage personnel de l'Apôtre (Conlationes VI 9, 2).

${ }^{21}$ RB 4, 53-54, SCh 181, 460 = Regula magistri [= RM] 3, 59-60, éd A. de Vogüé, t. I-II, SCh 105-106, Paris 1964, ici 105, 370.

${ }^{22}$ RB 7, 59, SCh 181, 486 = RM 10, 78, SCh 105, 436, citant Si 21, 23; cfr. Joannes Cassianus, De institutis coenobiorum IV 39, 2.

${ }^{23}$ Cfr. Lc 6, 25, cité par Basilius, Regulae 8, 30-31; Salvianus Massiliensis, De gubernatione VI 29; Isidorus, Sententiae III 20, 2.

${ }^{24}$ Outre Basile et Salvien (n. 23), voir Ferreolus, Regula ad monachos 24, 2-3. Pleurs de Jésus: Lc 19, 41-44; Jn 11, 35; He 5, 7. 
prière du carême s'accompagne de „larmes” et de „componction du coeur”, aussi bien que d'abstinence ${ }^{25}$. Loin d'empêcher la joie, ces manifestations de douleur spirituelle en sont l'aliment.

3. Plénitude de la joie chez saint Jean. La joie qui naît de l'épreuve et de la souffrance: cette séquence que présente la Règle bénédictine et que nous avons retrouvée chez saint Paul, on la trouve aussi dans un passage de saint Jean. Quand Jésus, à la dernière Cène, parle de son départ prochain et de sa séparation d'avec ses disciples, ceux-ci en éprouvent de la tristesse ${ }^{26}$, et Jésus leur prédit qu'ils vont „pleurer et se lamenter”, tandis que le monde se réjouira. Mais cette tristesse, ajoute-t-il, se changera en joie. Et pour illustrer sa prédiction, le Christ évoque la femme en travail dans les douleurs de l'enfantement, dont la tristesse disparaît à la naissance de l'enfant et se transforme en joie ${ }^{27}$. „Vous de même, conclut-il, vous éprouvez de la tristesse à présent. Mais je vous reverrai, et votre coeur se remplira de joie, d'une joie que personne ne pourra vous ôter" 28 .

Avant et après ce passage central, qui fait penser à la joie dans l'épreuve chez saint Benoît, le Quatrième Evangile parle plusieurs fois de la joie, en des termes qui reviennent avec une insistance frappante. Le verbe qui accompagne

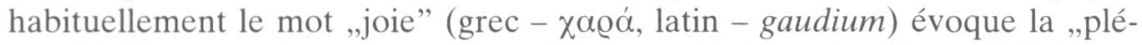
nitude" de ce sentiment dans l'âme de ceux qui aiment le Christ. Déjà JeanBaptiste, l'ami que réjouit le voix de l'Époux, dit que cette joie qu'il éprouve est „plénière”29. Et le Christ, à son tour, emploie plusieurs fois le même terme: „Je vous ai dit ces choses pour que ma joie soit en vous et que votre joie soit plénière" ${ }^{30}$; „demandez et vous recevrez, afin que votre joie soit plénière" 31 ; ,je dis cela dans le monde pour qu'ils aient ma joie en eux dans sa plénitude" ${ }^{\text {, }}$.

Deux autres fois, dans ses Lettres, Jean emploie le même vocabulaire: „Nous vous écrivons cela pour que vous vous réjouissiez et que votre joie soit plénière" 33 ; ,j'espère me retrouver chez vous et vous parler face à face, pour que vous ayez la joie en piénitude" 34 . Et à trois reprises, le Quatrième Evangile parle encore de la joie, soit à propos de ceux qui sèment et de ceux qui moissonnent ${ }^{35}$, soit au sujet d'Abraham et de la connaissance qu'il a eue du

${ }^{25}$ Cfr. RB 49, 4-7.

${ }^{26}$ Cfr. Jn 16, 6: ,tristitia implevit cor vestrum”.

${ }^{27}$ Cfr. Jn 16, 20-21.

28 Cfr. Jn 16, 22.

29 Cfr. Jn 3, 29: „Hoc ergo gaudium meum impletum est (grec - $\pi \varepsilon \pi \lambda \eta ́ \varrho \omega \tau \alpha \iota)$.

30 Jn 15, 11 (latin - impleatur; grec - $\pi \lambda \eta \varrho \omega \theta \tilde{\eta})$.

31 Jn 16, 24 (latin - plenum; grec - $\pi \varepsilon \pi \lambda \eta \varrho \omega \mu \varepsilon \dot{v} \eta$ ).

32 Jn 17, 13 (latin - impletum; grec - $\pi \varepsilon \pi \lambda \eta \varrho \omega \mu \varepsilon ́ v \eta v)$.

${ }^{33}$ 1Jn 1, 4 (latin - plenum; grec - $\left.\pi \varepsilon \pi \lambda \eta \varrho \mu \varepsilon ́ v \eta\right)$.

${ }^{34} 2$ Jn 12 (latin - plenum; grec - $\left.\pi \varepsilon \pi \lambda \eta \varrho \mu \varepsilon \dot{v} \eta\right)$.

35 Cfr. Jn 4, 36: „ut et qui seminat simul gaudeat et qui metit”. 
Christ $^{36}$, soit en une phrase où le Christ lui-même dit sa joie de prévoir que ses disciples vont croire en lui ${ }^{37}$.

4. Joie et „liesse” chez le Maître. Quant on a retrouvé dans l'Ecriture, source majeure de la pensée de Benoît, ce que l'Esprit Saint a dit de la joie, il n'est pas inutile d'écouter aussi les propos d'un auteur très proche de Benoît si proche qu'on peut se demander si ce „Maître” anonyme n'est pas Benoît luimême en son jeune temps. La Règle du Maître a en tout cas précédé la Règle bénédictine, qui reproduit ou adapte une grande partie de sa substance ${ }^{38}$.

Trois fois plus longue que celle de Benoît, l'oeuvre du Maître a aussi, en ce qui concerne la joie, un vocabulaire plus varié: aux mots gaudere et gaudium, seuls employés par Benoît, le Maître ajoute le verbe laetari (7 fois), le substantif laetitia (13 fois) et l'adjectif laetus (1 fois). A la différence de gaudere et de gaudium, d'où viennent nos mots français ,,jouir” et ,joie”, la laetitia n'est représentée dans notre langue que par un mot d'emploi très limité: „liesse”.

Que dit donc la Règle du Maître de cette ,joie” et de cette „liesse”? La première apparaît dès le début de la Règle, quand le Maître évoque, dans son Thema (Introduction), ce qui est à l'origine de toute vie monastique: l'eau du baptême. Après avoir bu à cette source divine, écrit-il, ,nous sommes restés là, stupéfaits, tant notre joie était grande "39. Cette joie si grande, voire „,excessive” (nimio), de la première rencontre avec Dieu reparaît à propos du Temps pascal: „Ceux qui sèment dans les larmes moissonneront dans la joie”, écrit le Maître, citant un Psaume ${ }^{40}$. Cette citation psalmique offre l'intérêt d'impliquer une conception du carême qui contraste avec celle de Benoît. Celui-ci, nous l'avons vu, représente ces quarante jours comme un temps où la „componction du coeur” fait „prier avec larmes”, certes, mais où toute la pénitence qu'on s'impose est „offerte à Dieu avec la joie du Saint Esprit” et l'on attend la sainte Pâque ,,avec la joie du désir spirituel”. De façon plus sommaire, le Maître oppose purement et simplement les larmes du carême à la joie de Pâques, qui apparaît comme un renversement complet de la tristesse précédente.

Entre cette première et cette dernière mention du gaudium, l'une à propos du baptême, l'autre au sujet du temps pascal, le Maître note encore que la joie accompagne l'hospitalité - on se réjouit de recevoir le Seigneur en la personne de l'hôte ${ }^{41}$ - et qu'elle caractérise notre sort éternel, en contraste avec les larmes

\footnotetext{
${ }^{36}$ Cfr. Jn 8, 56: „Abraham vidit et gavisus est”.

37 Cfr. Jn 11, 15: „gaudeo propter vos ut credatis”.

${ }^{38}$ Nous citons notre édition de La Règle du Maître, cfr. n. 21.

39 RM Th 12, SCh 105, 298: „stetimus stupidi nimio gaudio”.

${ }^{40}$ Cfr. RM 53, 20, SCh 106, 246: „Qui seminant in lacrimis in gaudio metent (Ps 125, 5).

${ }^{41}$ Cfr. RM 1, 16, SCh 105, 332: „pro gaudio supervenientis”.
} 
d'ici-bas ${ }^{42}$. Mais la plus appréciable de ces mentions de la joie est peut-être celle que fait le Maître en citant plusieurs des „fruits de l'Esprit” énumérés par saint Paul. Sa liste des „outils spirituels avec lesquels on exerce l'art divin” commence en effet par les trois vertus théologales - pauliniennes elles aussi - de foi, espérance et charité, mais il ajoute aussitôt: „la paix, la joie, la douceur” ${ }^{43}$, que suit la triade des grandes vertus monastiques: „l'humilité, l'obéissance, la taciturnité”.

Caritas, pax, gaudium, mansuetudo: moyennant une interversion - Paul mettait la joie avant la paix - ce sont là les quatre premiers „fruits de l'Esprit" énumérés par l'Apôtre aux Galates ${ }^{44}$. Après la charité (agapè, caritas), troisième des vertus théologales et premier des fruits de l'Esprit, voici donc la joie (grec- $\chi \alpha \varrho \alpha$, latin-gaudium) rangée par mi ces derniers et proposée aux moines, à ce titre, comme un „outil spirituel” de grande importance. Le moine doit donc cultiver la paix et la joie, aussi bien que la foi, l'espérance et la charité.

Quand on passe de gaudium à l'autre terme désignant la joie chez le Maître - laetitia - il faut d'abord remarquer la fréquence accrue des mots de cette famille: aux onze emplois de gaudium, gaudere et gauesci ${ }^{45}$ répondent les vingt occurrences de laetari, laetitia et laetus. Ce dernier adjectif n'apparaît qu'une fois, à propos de l'alleluia pascal ${ }^{46}$, mais le substantif laetitia (,liesse”) ne revient pas moins de onze fois, soit à propos de la même joie de Pâques ${ }^{47}$, soit en parlant de la joie éternelle que celle-ci préfigure ${ }^{48}$, soit encore à l'occasion du dimanche, jour de la Résurrection, et d'autres fêtes ${ }^{49}$, soit enfin à propos de la visite d'un frère spirituel, en qui l'on reconnaît le Seigneur luimême $e^{50}$. Quant au verbe laetari, ses sept emplois sont provoqués par les mêmes causes: liesse de Pâques et de $\mathrm{Nol}^{51}$, liesse dominicale ${ }^{52}$, liesse de l'éternité ${ }^{53}$.

${ }^{42}$ Cfr. RM 11, 78, SCh 106, 24, citant de nouveau Ps 125, 5 (selon le Psautier Romain, tandis que la Vulgate donne exultatione au lieu de gaudio).

${ }^{43}$ Cfr. RM 4, 1, SCh 105, 376 (fides, spes, caritas) et 4, 2 (pax, gaudium, mansuetudo). La charité sert de liaison entre les deux listes.

${ }^{44}$ Ga 5, 22-23, où le quatrième terme (grec - $\mu \alpha$ roo $\theta v \mu i \alpha$ ) est rendu par patientia dans la Vulgate, qui emploie mansuetudo pour traduire un autre mot.

45 Cfr. RM 81, 15 et 82,29, SCh 106, 332 et 340. Ce verbe est un mot tres rare et quelque peu obscur.

46 Cfr. RM 53, 49: „nova laetae resurectionis alleluia”; voir 3, 93 (n. 47).

${ }^{47}$ Cfr. RM 3, 93, SCh 105, 374: ,laetitiae canticum [...] alleluia”; 28, 44, SCh 106, 158: laetitiae alleluia".

48 Cfr. RM 10, 93, SCh 105, 440: ,in sempiternae laetitiae exultatione”; 10, 116; 53, 22; 90, 16.

49 Cfr. RM 27, 45, SCh 106, 148: „propter caritatem laetitiae sanctorum dierum” (dimanches et fêtes); 45, 7 ( Nol) et 18 (dédicace de 1'oratoire).

50 Cfr. RM 61, 5, SCh 106, 280: „pro adventus tui laetitia”.

51 Cfr. RM 53, 19, SCh 106, 246: „laetari cum Christo”; 45, 7 (Nol et Pâques).

52 Cfr. RM 45, 14, SCh 106, 208, citant Ps 149, 5: „laetabuntur in cubilibus suis (les saints dont on conserve les reliques dans les églises). Ce verset psalmique est répété aux offices du dimanche, jour de repos et de liesse, jusqu'a la messe $(75,7)$. On le dit aussi à chaque visite d'église $(57,26)$.

53 Cfr. RH 53, 25, SCh 106, 246: „cum eo [...] in futuro laetari”. 
5. Joie et tristesse dans la Vie de Benoît. Autant la Règle du Maître, source littéraire de celle de saint Benoît, abonde en notations relatives à la joie, autant le Deuxième Livre des Dialogues de Grégoire le Grand, qui raconte la vie du saint, parle peu de gaudium ou de laetitia. Ti faut attendre un des derniers chapitres pour voir Benoît "se réjouir” de la fin glorieuse de sa soeur Scholastique, dont il a vu l'âme entrer au ciel sous la figure d'une colombe. Cette joie spirituelle le fait éclater en hymnes de louange et d'action de grâces ${ }^{54}$. Au reste, cette fin si heureuse a été précédée, trois jours plus tôt, par un long entretien nocturne où le frère et la soeur ont parlé ensemble des ,joies de la vie céleste" ${ }^{25}$. Ce n'est donc pas seulement ici-bas, mais aussi et surtout dans l'au-delà que se trouve la joie, objet d'espérance.

La même orientation eschatologique apparaît dans l'épisode qui suit. Aussitôt après, en effet, Grégoire parle des rapports du saint avec un autre abbé qui lui ressemblait, ce diacre Servandus dont le monastère se trouvait à Naples et qui venait souvent le voir. Ces fréquentes visites avaient un but tout spirituel: comme Benoît, Servandus était:

„,imprégné de doctrine et de grâce céleste. C'était entre eux une transfusion de douces paroles de vie. La suave nourriture de la céleste patrie, ils ne pouvaient encore en jouir parfaitement; du moins ils en avaient un goût en soupirant vers elle" ${ }^{n 6}$.

Gaudendo: ce mot que la traduction française rend par ,jouir”, c'est le verbe qui dit la ,,joie”. Celle-ci, sous sa forme ,parfaite” est réservée à l'au-delà. Icibas, les joies du ciel ne sont „goûtées” qu'en „soupirant”.

Scholastique et Servandus: ces deux personnes, à propos desquelles le biographe parle de joie, n'apparaissent qu'à la fin du récit, quand Benoît s'apprête à passer le cap de la mort et à connaître les joies de l'éternité. Mais dans les pages précédentes on le voit, à deux reprises, consoler des âmes endolories. A Subiaco, quand il a repêché l'outil qu'un moine goth avait laissé tomber dans le lac, il dit à ce disciple affligé: „Travaille et ne t'attriste pas”, ${ }^{\text {, }}$. Et quand, au Mont-Cassin, une famine afflige les frères, Benoît promet à ceux-ci un secours de la Providence en leur disant:

„Pourquoi vous attrister pour un manque de pain? Aujourd'hui c'est la disette, demain ce sera l'abondance" 58 .

${ }^{54}$ Cfr. Gregorius Magnus, Dialogi II 34, 1, éd A. de Vogüé, SCh 260, 234: „Qui tantae eius gloriae congaudens, omnipotenti Deo in hymnis et laudibus gratias egit".

55 Ibidem II 33, 2, SCh 260, 230: „ut usque mane de caelestis vitae gaudiis loquamur”.

56 Ibidem II 35, 1, SCh 260, 236: „ut, quia isdem quoqne vir doctrina gratiae caelestis influebat, dulcia sibi invicem vitae verba transfunderent, et suavem cibum caelestis patriae, quia adhuc perfecte gaudendo non poterant, saltem suspirando gustarent.

57 Ibidem II 6, 2, SCh 260, 156: „Ecce labora et noli contristari”.

58 Ibidem II 21, 1, SCh 260, 198: „Quare de panis inopia vester animus contristatur? Hodie quidem minus est, sed die crastina abundanter habebitis”. 
Malgré ces efforts pour dissiper la tristesse d'autrui, Benoît est assez souvent attristé lui-même par des faits malheureux. La haine acharnée que lui voue, à Subiaco, le curé du lieu, le fait souffrir ${ }^{59}$. Et quand ce mauvais prêtre disparaît brusquement, Benoît se lamente sur son sort, ainsi que sur la joie inconsidérée que Maur a tirée de ce malheur ${ }^{60}$.

Au Mont-Cassin, on voit de nouveau le saint exaspéré par un de ses moines qui l'assaille de ses plaintes et auquel il finit par dire de s'en aller ${ }^{61}$. Plus loin Grégoire montre, à deux reprises, Benoît „,contristé” par des requêtes ou des agissements de gens du dehors: un père qui lui demande de ressusciter son enfant mort; sa propre soeur Scholastique qui l'oblige à passer la nuit hors du monastère ${ }^{62}$

Au total, la Vie de Benoît présente une image de saint très humaine et vraie, où la tristesse n'est pas moins présente que la joie, celle-ci étant surtout l'objet d'une grande espérance.

6. Le vice de tristesse chez Cassien. Au-delà de la Règle du Maître, et tant à travers celle-ci que directement, Benoît tient sa doctrine spirituelle du grand maître des moines occidentaux que fut Jean Cassien. Selon celui-ci, la tristesse est un des huit vices principaux contre lesquels les moines, ainsi que tous les chrétiens, ont à lutter sans cesse. Les solitaires, en particulier, sont souvent et gravement atteints de tristesse ou d'acédie, note l'auteur des Conférences ${ }^{63}$, et ces deux vices sont d'autant plus difficiles à combattre que, à la différence des autres, ils ne sont pas toujours provoqués par des causes externes, mais parfois par des mouvements intérieurs ${ }^{64}$. En d'autres termes, la tristesse survient souvent sans raison apparente, de sorte qu'on ne sait que faire pour la chasser.

Si la tristesse est un vice, la joie, qui est son opposé, serait-elle une vertu? Cassien n'a jamais dit cela, à notre connaissance, mais il ne manque pas de mentionner la joie parmi les „fruits de l'Esprit” énumérés par saint Paul, qui sont „charité, joie, paix” et le reste ${ }^{65}$. L'occasion et le contexte de cette citation paulinienne sont d'ailleurs pleins d'intérêt. Traitant là du vice de tristesse, l'auteur des Institutions se souvient d'une autre parole de l'Apôtre: la distinction entre la „tristesse selon Dieu”, qui conduit à la pénitence

${ }^{59}$ Cfr. ibidem II 8, 4, SCh 260, 162: ,illi magis quam sibi doluit”.

${ }^{60}$ Cfr. ibidem II 8, 7, SCh 260, 164: ,sese in gravibus lamentis dedit”.

${ }^{61}$ Cfr. ibidem II 25, 1,SCh 260, 212: „nimietatis eius taedio affectus, iratus iussit ut discederet".

62 Cfr. ibidem II 32, 2, SCh 260, 228: „valde contristatus est”; 33, 4. SCh 260, 232: „coepit conqueri contristatus".

${ }^{63}$ Cfr. Joannes Cassianus, Conlationes V 9, éd. E. Pichery, SCh 42, 197.

${ }^{64}$ Cfr. ibidem V 3, SCh 42, 190.

${ }^{65}$ Cfr. idem, De institutis coenobiorum IX 11, éd. J.C. Guy, SCh 109, 376, citant Ga 5, 22-23. 
et au salut, et la „,tristesse du siècle”, qui produit la mort ${ }^{66}$. La tristesse selon Dieu par laquelle on se repent du péché, dit Cassien, s'accompagne d'une effusion de l'Esprit qui comporte la joie, tandis que la tristesse du siècle est privée de ces dons spirituels. Paradoxalement, on peut dire de la tristesse selon Dieu qu'elle est ,joyeuse, en quelque sorte, et animée de l'espoir de progresser" ${ }^{\circ 7}$.

Ce paradoxe d'une tristesse non dépourvue de joie, d'une tristesse qui engendre même une certaine joie, est au coeur de la vie monastique. Comment ne pas se rappeler, à ce propos, que Jésus lui-même a „ressenti tristesse et angoisse”, avouant qu'il avait „l'âme triste à en mourir"” ${ }^{68}$. Ces paroles de l'Evangile, que Cassien ne cite jamais ${ }^{69}$, sont bien faites pour nous rassurer à l'heure des épreuves. Mais l'opposition paulinienne des deux tristesses, que fait valoir Cassien, est aussi une grande lumière, apte à prémunir le moine contre la tristesse vicieuse, stérile, contraire à l'Esprit.

7. Trois Règles font espérer la joie. Dans la famille nombreuse des Règles monastiques, il en est trois qui citent, chacune en un contexte particulier, la parole répétée du Christ dans la parabole des serviteurs: „Entre dans la joie de ton maître" (Mt 25, 21-23).

Dans les années 553-581, Ferréol, évêque d'Uzès, invite les moines d'un monastère qu'il a fondé hors de son diocèse, à révérer et aimer leur abbé. Grande, en effet, est la responsabilité de celui-ci. Ayant beaucoup reçu, il devra rendre compte de tout cela. Puisse-t-il alors s'entendre dire: „C'est bien, serviteur bon et fidèle, entre dans la joie de ton maître!"70.

Un peu plus tard sans doute, vers 580, Léandre de Séville adresse à sa jeune soeur Florentine, consacrée à Dieu depuis l'enfance, une longue exhortation à demeurer dans sa communauté de vierges et à y profiter au maximum des biens spirituels dont elle est comblée. Curieusement, c'est dans une mise en garde contre le rire que retentit l'appel à la joie:

„Que ton âme, dans la joie de Dieu, soit remplie d'une allégresse tranquille et modérée, selon la parole de l'Apôtre: «Soyez toujours joyeux dans le Seigneur. Je le répète: soyez joyeux» $(\mathrm{Ph} 4,4)$. Et ailleurs il dit: «Le fruit de l'Esprit, c'est la joie» $(G a 5,22)$. Cette allégresse-là ne trouble pas les facultés mentales en les

${ }^{66}$ Cfr. ibidem IX 10, SCh 109, 376, citant 2Co 7, 10: caritas, gaudium, pax, etc.

${ }^{67}$ Ibidem IX 11, SCh 109, 378: „quodammodo laeta et spe profectus sui vegeta”.

${ }^{68}$ Cfr. Mt 26, 37-38; Mc 14, 34. Voir aussi Lc 22, 43-44; Jn 12, 27. Dès avant sa Passion, on voit Jésus „navré” de l'endurcissement de certains auditeurs (Mc 3,5).

${ }^{69}$ Seul Lc 22, 44 est cité dans Conlationes IX 25, où ces ,gouttes de sang” du Christ à Gethsemani sont évoquées comme les signes d'une prière sublime, indicible (la „prière de feu").

${ }^{70}$ Ferreolus, Regula ad monachos 2, 6, PL 66, 961A. 
salissant par le rire, mais elle élève l'âme et lui fait désirer le repos céleste, où il lui sera donné d'entendre ces mots: «Entre dans la joie de ton maître»"71.

Cette fois, la joie n'apparaît pas seulement comme une récompense dans l'audelà, mais comme un don de l'Esprit qui comble l'âme dès à présent. L'espérance devient expérience, celle-ci précédant et suscitant celle-là. Cette joie qui vient de Dieu n'a que faire du rire, dont Léandre fait le procès dans les lignes suivantes, allant jusqu'à préconiser les larmes et les soupirs de l'âme qui aspire à ,être avec le Christ". Paradoxalement ce chapitre, qui commence par préconiser la joie, s'achève ainsi par une invitation à pleurer. Mais le dernier mot est pour la consolation: „Si tu pleures à cause de son absence, il te consolera par sa présence".

Une soixantaine d'années plus tard, vers 640, un troisième évêque, Donat de Besançon, écrit une Règle pour les soeurs du monastère qu'ont fondé ses parents. Commençant, comme Benoît, par un directoire de l'abbesse, il termine ce premier chapitre en faisant espérer à la supérieure qu'elle entendra un jour le Seigneur lui dire, comme aux bons et fidèles serviteurs: „Entre dans la joie de ton maître” ${ }^{, 72}$. Cette fois, on retrouve le contexte purement eschatologique dans lequel l'Evangile situe cette parole. Comme Ferréol, Donat pense seulement à la joie finale de l'au-delà. C'est donc chez Léandre seul qu'apparaît une anticipation de cette joie céleste, qui n'exclut nullement, d'ailleurs, les souffrances terrestres, mais au contraire engendre une certaine douleur, dans l'attente de la joie pure et plénière qui nous est promise.

8. Une joie perpétuelle: pourquoi pas? Dans cette perspective évangélique, la joie apparaît comme un bien futur, comme une promesse pour l'au-delà. Mais qu'en est-il de la vie présente? A cet égard, nous pouvons nous remémorer le précepte donné par saint Paul aux Thessaloniciens: „Soyez toujours joyeux". Mais il faut avouer que ce commandement paulinien n'a peut-être pas reçu, dans la tradition chrétienne, toute l'attention qu'il mérite. Déjà nous avons reconnu ce fait en comparant le „Soyez toujours joyeux” à la parole qui le suit immédiatement: „Priez sans cesse”. Alors que celle-ci a provoqué l'immense effort des moines pour pratiquer la prière incessante, soit sous la forme collective de l'office divin, soit par les multiples réalisations individuelles d'un chacun, on n'entend point parler d'une doctrine ou d'une pratique similaire en vue de cultiver toujours la joie.

Cette remarque que nous avons faite plus haut à propos de la prière, nous pouvons l'étendre maintenant au dernier élément de la triade paulinienne:

${ }^{71}$ Leander Hispalensis, Regula 21, PL 72, 886.

72 Donatus Vesuntinus, Regula ad virgines 1, 22 corrigeant opportunément RB 64, 22. 
l'action de grâces. "Soyez toujours joyeux, priez sans cesse, en toute chose rendez grâces”. L'eucharistie ou „,action de grâces”, elle aussi, a été l'objet d'un effort de perpétuité, qui s'exprime notamment dans la formule solennelle de la liturgie: „Oui, il est vraiment digne et juste, équitable et salutaire, que nous te rendions grâces toujours et partout, à toi, Seigneur saint, Père toutpuissant, Dieu éternel [...]”. Ces mots, que le prêtre répète à chaque messe, vérifient exactement le précepte de saint Paul: „En toute chose, rendez grâces”. De même que le „priez sans cesse” se déploie dans 1',oeuvre de Dieu” des moines et la prière quotidienne des fidèles, de même le „Rendez grâces en toute chose" s'exprime dans cette eucharistie qui est l'acte central et sans cesse renouvelé de l'Eglise entière.

Ce constat nous incite à poser la question: pourquoi le premier terme de la trilogie paulinienne ne susciterait-il pas un effort analogue de notre part ? Pourquoi le „Soyez toujours joyeux" ne serait-il pas pris au sérieux, comme les deux injonctions qui le suivent? Pourquoi notre joie ne serait-elle pas incessante, tout comme notre prière et notre action de grâces? Spe gaudentes: ,joyeux dans l'espérance". Si nous ne cultivons pas la joie aussi bien que ses deux compagnes, n'est-ce pas parce que notre espérance est trop faible, notre désir de la vie éternelle trop peu ardent, notre regard trop absorbé par des objets terrestres voués à périr?

Dieu nous appelle à la joie éternelle, et dès ici-bas, dans l'espérance, nous pouvons et devons cultiver la joie. Pour fortifier notre conviction à cet égard, nous pouvons nous rappeler deux passages de l'Epître aux Philippiens où saint Paul réunit de nouveau la joie, la prière et l'action de grâces, comme il l'avait fait, quelques années plus tôt, dans sa lettre aux chrétiens de Thessalonique. Cette Epître aux Philippiens parle de la joie dans une douzaine de passages, et la chose est d'autant plus remarquable que Paul est alors prisonnier et va vers sa fin. Sans passer en revue toutes ces mentions de la joie, bornons-nous aux deux phrases qui associent la joie à ses deux soeurs. Voici la première, qui suit la salutation initiale: ,Je rends grâces à mon Dieu toutes les fois que je me souviens de vous, dans toutes mes prières, priant pour vous tous avec joie" 73 .

Dans la lettre aux Thessaloniciens, Paul rangeait ces trois actes dans l'ordre suivant: joie, prière, action de grâces. Ici, il renverse cet ordre: l'action de grâces vient en tête, suivie de la prière et de la joie. Mais les deux triades sont substantiellement identiques, et la note de perpétuité (,,toutes les fois[...] dans toutes mes prières") retentit maintenant comme par le passé.

Commencée de la sorte, la lettre aux Philippiens se termine de même, et cette fois il s'agit d'une phrase que la liturgie nous a rendue familière: „Soyez

${ }^{73} \mathrm{Ph} \mathrm{1,3-4:} \mathrm{„Gratias} \mathrm{ago} \mathrm{Deo} \mathrm{meo} \mathrm{in} \mathrm{omni} \mathrm{memoria} \mathrm{vestri,} \mathrm{semper} \mathrm{in} \mathrm{cunctis} \mathrm{orationibns} \mathrm{meis}$ pro omnibus vobis cum gaudio deprecationem faciens". 
toujours joyeux dans le Seigneur; je le répète: Soyez joyeux. Que votre bonne conduite apparaisse à tous les hommes. Le Seigneur est proche. N'ayez aucun souci, mais qu'en toute chose, par une prière et une supplication accompagnées d'action de grâces, vos demandes se fassent connaître à Dieu"74.

Joie, prière, action de grâces: cette fois, les trois termes sont rangés dans le même ordre que jadis, quand saint Paul s'adresssait aux fidèles de Thessalonique. Et cet ordre met en relief la joie, nommée la première et expressément prescrite „toujours”, avec une répétition qui en souligne la nécessité. On ne peut être plus formel sur ce devoir d'être joyeux sans cesse.

${ }^{74} \mathrm{Ph}$ 4, 4-6: „Gaudete in Domino semper, iterum dico: gaudete. Modestia vestra nota sit omnibus hominibus. Dominus prope est. Nihil solliciti sitis, sed in omni oratione et obsecratione cum gratiarum actione petitiones vestrae innotescant apud Deum”. 